\title{
A Fast Context -Based Fractal Mobile Video Compression with GA and PSO
}

\author{
Junshe Wan \\ Department of Automatic Control \\ South China University of Technology \\ GuangZhou, 410072, China \\ e-mail:jun_she@163.com
}

\author{
Linru You \\ Department of Automatic Control \\ South China University of Technology \\ GuangZhou, 410072, China \\ e-mail:aulryou@scut.edu.cn
}

\begin{abstract}
Mobile video compression is an important method to translate multimedia from server to client, especially mobile video compress with fractal model is a good way to solution the unbalance computer capacity. Because computer capacity of servers are so powerful that complete encode of fractal video compress for server-end, comparing the mobile phone is so poor that decode of fractal video compress for client-end. In this paper, basing on the segment, we present GA to improve the quality of video compress through adjust the fixed parameter to suitable value in fractal compress algorithm. For improving speed, the paper describes PSO to speed up the fractal video compress. PSO method could find the best domain when we face huge samples to search. Compare the GA method, as it does not need the crossover and mutation, the speed of convergence is clear faster when we use PSO method to find the best G. Another issue is that we couldn't ensure that the new crossover and mutation must follow the field the original Domains. If that, we should add more samples to domains. Experiments shows that the PSO could better match the requirement of searching the best domain comparing GA method on the premise of guaranteeing the decoded image's quality.
\end{abstract}

Keywords- fractal video compression; image segmentation; $G A ; P S O$

\section{INTRODUCTION}

Mobile video application is more and more widespread through telecom method adjust from $2 \mathrm{~g}$ to $3 \mathrm{~g}$ even more $4 \mathrm{~g}$ ages. Comparing computing capacity between clouding server and mobile phone, we cloud know that the clouding server is so powerful and mobile phone is so poor. The unbalance is suitable to fractal video compress algorithm. The algorithm of video compression, which is used by the MPEG standards, will be elucidated upon in order to explain video compression [1]. However the general mobile video meet the requirement of H.264 format [2], comparing with PC with MPEG-4 format. MPEG-4 is a very low bit rate video in the T. Sikor's paper [3]. Over the past decades, Image and Video Compression for Multimedia technologies have some people liking Yun Q.Shi and Huifang Sun present MPEG general method [4]. Yao Wand, Jorn Ostermann and Ya-Qin Zhang also described how to down cost of communication [5].However network client, their algorithms are so good that quality is keep the original, namely compress, it require that the mo we firstly discuss the application of fractal video compress, which is based the theory if iterated function system (IFS) described in $[6,7]$.
For hard ware method is found in [8, 9, 10]. In [8], the authors propose hardware/software code sign methodology for image compression using an FPGA. They show a speedup of $6 \mathrm{x}-8 \mathrm{x}$ vs. Software In [9], acceleration using GPUs was considered; however the work is limited to approximately1 frame per second and is nowhere near realtime. Other works such as [10] have shown encoding for videos with low resolution and quality.

Particle swarm optimization (PSO) was originally introduced by Kennedy and Eberhart in 1995 [11]. It is based on the social behaviors of bird flocking or fish schooling. But last years PSO has been turned out to be another powerful tool besides other evolutionary algorithms such as genetic algorithms (GAs) [12]. However, the performance of PSO is decided mostly to its control parameters, liking inertia weight $\mathrm{w}$ and learning factors, $\mathrm{c} 1$ and $\mathrm{c} 2$. These parameters determine the performance of PSO, and slightly different settings may lead to very different performance. Whether PSO or GA is chose by your real applications of different requirements. In the past several years, some researchers have proposed different approaches to find a good set of parameters. Shi and Eberahrt [13] introduced a parameter called inertia weight $\mathrm{w}$ for the original PSO algorithm. The inertial weight is used to balance the global and local search abilities. A large inertia weights suitable for global search, and a small inertia weight is beneficial for local search. A linearly decreasing inertial weight over the course of search achieves good performance. Clerc and Kennedy [14] proposed a constriction factor in to PSO, which guarantees the convergence and improves the convergence velocity. Secrest and Lamont [15] modified the velocity updating model and proposed a Gaussian model, in which the learning factors are replaced with random values based on Gaussian distribution. Based on graph context, the paper [16] proposed a fast image segmentation algorithm, by comparing the characteristic difference between regions and interior domains, it can judge whether there is a boundary between the two regions. In order to improve quality of compress, Raouf Hamzaoui, Dietmar Saupe, and Michael Hiller [17] present some effective methods to reduce the Distortion on the premise of guaranteeing the decoded image's quality. In order to improve the speed, the papers $[22,23,24$, and 25] present some invaluable methods to refer.

In this paper, we describe both encode and decode of fractal video compress are together described clearly with codebook method. In order to improve quality, the GA is adopted to improve the quality of whole compress. In the 
same time, how to speedup of compress is used to PSO. Through the experiment we show that the GA of fractal algorithm could improve the quality of mobile video compress, and PSO of fractal algorithm could speed up the whole mobile video compress. As you know that the GA and PSO are belong to the evolutionary computation.

The rest of this paper is organized as follows: section 2 introduces some basic theory about fractal image compression, and following in section 3, we discuss some typical GA and PSO approaches; we propose our approach to improve quality and speed up encode. To evaluate the quality and speed of our adapted approach, comparison experiments are conducted in section 4; Section 5 concludes this paper.

\section{SEGMENT OF FRACTAL VIDEO COMPRESS}

Suppose that we have a $1024 * 1024$ image, the basic ideal of fractal image compression is as paper[18], Hai Wang divide initial image into small image blocks with nonoverlapping (Rang block, $\mathrm{R}$ block for short). For each $\mathrm{R}$ block, find an image block (Domain block, D block for short) which is the most similar to current $\mathrm{R}$ block under a certain transform, that is, use some image blocks' transformation to splice the initial image, and make the spliced image similar to the original image as much as possible. Now we choose $\mathrm{R}$ is $8 * 8$ as basic Range.

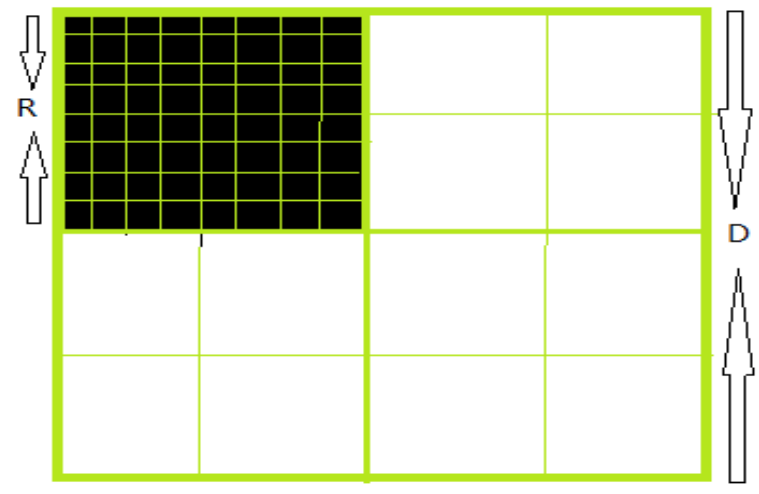

Figure 1. Example Rang and Domain

Algorithm of encodeing is liking steps as below: (R meansRange,D menas Domain)

$$
\begin{aligned}
& \text { Step } 1, \mathrm{R}=\left(\frac{1024}{8}\right)^{2}=16384 \\
& \text { Step } 2, D=\left(1024-2^{*} 8+1\right)^{2}=1018081 \\
& \text { Step } 3, D^{\prime}=\text { Average } D, 16 * 16 \longrightarrow 8 * 8 \\
& \text { Step } 4, \mathrm{D}_{8 x}{ }^{\prime}=D^{\prime} *\left[T_{x}\right]
\end{aligned}
$$

$$
\begin{aligned}
& \text { Step5, MSE }=\left\|\left(s_{k} * D_{k}+o_{k} * I-R_{k}\right)\right\| \\
& S_{k}=\frac{B^{2}<D_{k}, R_{k}>-<D_{k} I><R_{k}, I>}{B^{2}<D_{k}, D_{k}>-<D_{k}, I>^{2}} \\
& o_{k}=\frac{<R_{k}, I>-s_{k}<D_{k}, I>}{B^{2}} \\
& \text { Step } 6, W=\left\{D_{k}(x, \mathrm{y}), T_{x}, S_{k}, o_{k}\right\}
\end{aligned}
$$

Table 1. Transformation matrix

\begin{tabular}{|c|c|c|c|}
\hline$T_{0}=\left(\begin{array}{ll}1 & 0 \\
0 & 1\end{array}\right)$ & $T_{1}=\left(\begin{array}{ll}0 & 1 \\
-1 & 0\end{array}\right)$ & $T_{2}=\left(\begin{array}{ll}-1 & 0 \\
0 & -1\end{array}\right)$ & $T_{3}=\left(\begin{array}{ll}0 & -1 \\
1 & 0\end{array}\right)$ \\
\hline-7 & -7 & -7 & - \\
\hline$T_{4}=\left(\begin{array}{ll}-1 & 0 \\
0 & 1\end{array}\right)$ & $T_{5}=\left(\begin{array}{ll}1 & 0 \\
0 & -1\end{array}\right)$ & $T_{6}=\left(\begin{array}{ll}0 & -1 \\
-1 & 0\end{array}\right)$ & $T_{7}=\left(\begin{array}{ll}0 & 1 \\
1 & 0\end{array}\right)$ \\
\hline
\end{tabular}

In the step4, Tx is liking as Table 1.Which $\operatorname{Dk}(\mathrm{x}, \mathrm{y})$ represents the D' block's starting position, to represents the corresponding affine transformation matrix in the middle of Table1.

As to decoding of fractal video compress, according to fixed point theorem, this iteration process would converge to a fixed attractor, and this attractor is the decoding image. The fractal decoding process is iterating process for five times.

This fixed block is not good chose because some time we should clearly class the ranges to one image liking Fig1. In fact we could follow the context to segment the image to different ranges, however it is critical issue that we always create excessive segment if we don't study the context according a real image.

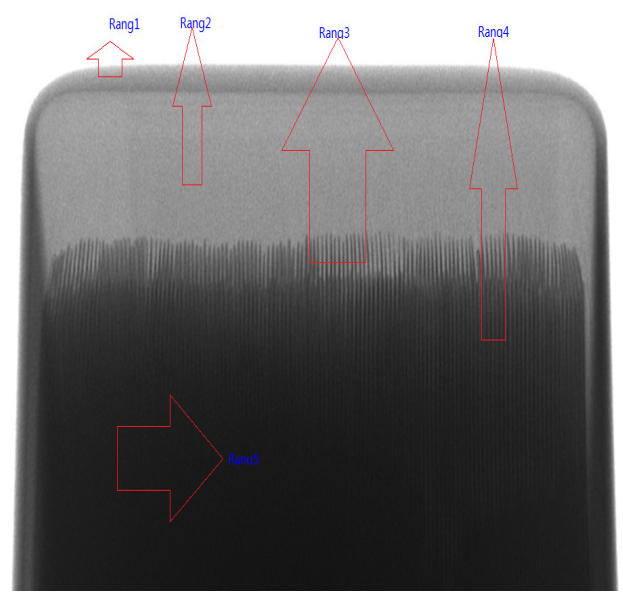

Figure 2. Five ranges of the battery through X-rays 
Through some methods have improve the encoding the quality, some new issues will emerge in the part of decoding. Especially different block encoding, what's rule that you ensure the order of decoding right is a huge challenge. In this paper, we present that we could use GA and PSO to parallel deal with choosing better, not best method to speed the encoding process.

\section{IMPROVE QUALITIES WITH EVOLUTIONARY COMPUTER}

Algorithm is started with a set of solutions (represented by chromosomes) called population. Solutions from one population are taken and used to form a new population. This is motivated by a hope, that the new population will be better than the old one. Solutions which are selected to form new solutions (offspring) are selected according to their fitness the more suitable they are the more chances they have to reproduce. The whole process could see in Fig3. Firstly we should accord the issue to encode, and then we could create some population to find a best or better result based on the Fitness Fun, if OK, the process is over, otherwise the next is choose, crossover, mutation to replace some old population.

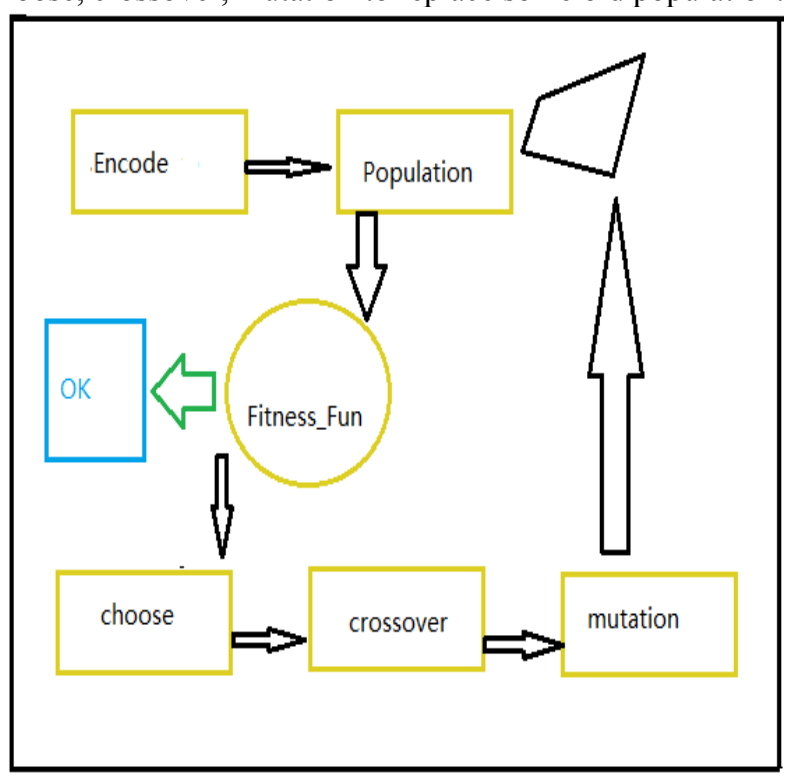

Figure 3. Example of GA process method

For finding best the Domain to match the requirement of Range, the GA process method is good to complete the task. The Domain is too big to find the best under limited time usually, how to solve the issue is follow two method, one is translate the computing with hardware liking DSP, GPU, FPGA, the other method is used AI algorithm to get a better value in the field of limited time. In this paper, we firstly use the GA to search the best the Domain to complete the encoding process of fractal compress. For the basic theory of PSO algorithm, we could see the velocity equation as below:

$v_{i}(k+1)=v_{i}(k)+\gamma_{1 i}\left(p_{i}-x_{i}(k)\right)+\gamma_{2 i}\left(\mathrm{G}-x_{i}(k)\right)$

Position equation :

$x_{i}(k+1)=x_{i}(k)+v_{i}(k+1)$
In the middle velocity and position equations, $\mathrm{i}$ mean particle index, $\mathrm{k}$ mean discrete time index, $\mathrm{v}$ mean velocity of ith particle, $x$ mean position of ith particle, $p$ mean best position found by ith particle(personal best), $G$ mean best position found by swarm(global best, best of personal bests),r mean random numbers on the interval[0,1] applied to ith particle. For the common PSO algorithm, we could describe as below:

$$
v_{i}(k+1)=\phi(k) v_{i}(k)+\alpha_{1}\left[\gamma_{1 i}\left(p_{i}-x_{i}(k)\right)\right]+\alpha_{2}\left[\gamma_{2 i}\left(\mathrm{G}-x_{i}(k)\right)\right]
$$

As training process, we should use decreasing linear inertia function, thus you ensure the result becomes smaller and smaller until to convergence.

In the fractal compress, the encoding spends $98 \%$ to find the best Domain in the huge number of Domains. For PSO, if we could find the $\mathrm{G}$ through per $\mathrm{Pi}$, the best object is $\mathrm{G}$. PSO method could find the best Domain when we face huge so big samples to search. Compare the GA method, as it does not need the crossover and mutation, the speed of convergence is clear faster when we use PSO method to find the best G. Another issue is that we couldn't ensure that the new crossover and mutation must follow the field the original Domains. If that, we should add more samples to Domains. Thus the PSO could better match the requirement of searching the best Domain comparing GA method.

Before you begin to format your paper, first write and save the content as a separate text file. Keep your text and graphic files separate until after the text has been formatted and styled. Do not use hard tabs, and limit use of hard returns to only one return at the end of a paragraph. Do not add any kind of pagination anywhere in the paper. Do not number text heads-the template will do that for you? The tradeoff of compress method must accord to data of Mean Square Value (MSE), Peak Signal to Noise Ratio (PSNR), Compression Ratio (CR), Encoding Time.

Table 2. Five methods compare result

\begin{tabular}{|c|c|c|c|c|}
\hline Approaches & MSE & Encoding & PSNR (dB) & CR \\
\hline PSO of Fractal & 70 & 2.9 & 35.1 & 9.1 \\
\hline GA of Fractal & 76 & 2.7 & 33.2 & 8.9 \\
\hline Reference[19] & 85 & 2.8 & 32.8 & 9.1 \\
\hline Reference[20] & 82 & 2.4 & 32.9 & 9.2 \\
\hline Reference[21] & 100 & 2.1 & 30.9 & 8.9 \\
\hline
\end{tabular}

According to data of Table2, we could find the PSO of fractal is the best result comparing [19],[20],[21],GA of Fractal. Both the quality and speed, the PSO of fractal is a good result in the all methods. Although PSO of Fractal of approach's encoding time is a little longer, it can significantly improve the recovered image's quality and compression ratio. So conclude all the above, compared with those typical approaches, PSO of Fractal of approach proposed in this paper has some advantages. 


\section{CONLUSION}

This paper briefly introduces the basic mobile video compare theory of image fractal compression, and discusses unbalance between upload and download is suitable typical fractal compression approaches. Based on fixed block fractal compression approach, we consider image's context, context-based image segmentation to fractal image compression, separating the initial image into many logic areas, and then encoding each area with fractal image compression method. Experimental results show that, compared with approaches proposed in reference [19] and [20], our proposed approach can shorten the encoding time significantly, and compared with approach proposed in reference [21], our proposed approach can improve the recovered image's quality and compression ratio significantly. But in this paper, we don't consider some other commonly used image processing technology such as RLE, wavelet transform and so on, so our further work is to apply some other commonly used image processing technology to fractal compression to further reduce the encoding time and increase the compression ratio.

\section{ACKNOWLEDGMENT}

The paper firstly thanks to company of Unicom to provide the studying condition to complete the whole study of mobile fractural compress code. And thanks Dr. of Xiaogang Chen to some suggestions about the papers.

\section{REFERENCES}

[1] CLARKE, R. J. Digital compression of still images and video. London: Academic press. 1995, pp. 285-299

[2] Iain E. G. Richardson, H.264 and MPEG-4 Video Compression, Video Codingfor Next-generation Multimedia, the Robert Gordon University, Aberdeen, UK,2003.

[3] T. Sikora, "MPEG-4 very low bit rate video," IEEE International Symposium on Circuits and Systems, ISCAS '97, vol. 2, pp. 14401443, 1997.

[4] Yun Q.Shi and Huifang Sun, "Image and Video Compression for Multimedia Engineering: Fundamentals, Algorithms, and Standards", CRC press, 2000.

[5] Yao Wand, Jorn Ostermann and Ya-Qin Zhang, "Video Processing and Communications", Prentice Hall, 2007.

[6] M. F. Barnsley, "Fractal Modelling of Real World Images," in Fractals Everywhere,2nd ed., 1993.

[7] Y. Fisher, Ed., Fractal Image Compression: Theory and Application. London, UK,UK: Springer-Verlag, 1995.

[8] O. Alvarado N and A. Daz P, "Acceleration of Fractal Image Compression Usingthe Hardware-Software Co-design Methodology," 2009 International Conference on Reconfigurable Computing and FPGAs, no. 2, pp. 167-171, 2009.
[9] U. Erra, "Toward Real Time Fractal Image Compression Using Graphics Hardware,"in International Symposium on Visual Computing 2005, vol. 3804, Lake Tahoe(Nevada), USA, 2005.

[10] D. Monro, J. A. Nicholls, C. Down, and B. Ay, "Real Time Fractal Video For Personal Communications," An Interdisciplinary Journal On The Complex Geometry of Nature, vol. 2, pp. 391-394, 1994.

[11] J. Kennedy and R. C. Eberhart. Particle swarm optimization. In Proceedings of IEEE International Conference on Neural Networks, pages 1942-1948, 1995.

[12] R. Eberhart and Y. Shi. Comparison between genetic algorithms and particle swarm optimization. In Proceedings of The 7th Annual Conference on Evolutionary Programming, 1998.

[13] Y. Shi and R.C. Eberhart. A modified particle swarm optimizer. In Proceedings of the Conference on Evolutionary Computation, pages 69-73, 1998

[14] M. Clerc and J. Kennedy. The particle swarm-explosion, stability, and convergence in a multidimensional complex space. IEEE Transactions on Evolutionary Computation, 6(1):58-73, 2002.

[15] B. Secrest and G. Lamont. Visualizing particle swarm optimizationgaussian particle swarm optimization. In Proceedings of IEEE Swarm Intelligence Symposium, pages 198-204, 2003.

[16] Pedro F. Felzenszwalb and Daniel P.Huttenlocher . Efficient GraphBased Image Segmentation.International Journal of Computer Vision, 59(2) September 2004

[17] Raouf Hamzaoui, Dietmar Saupe, and Michael Hiller. Distortion Minimization with Fast Local Searchfor Fractal Image Compression. Journal of Visual Communication and Image Representation 12,450468 (2001)

[18] Hai Wang, Fast Image Fractal Compression with Graph-Based Image Segmentation Algorithm, International Journal of Graphics Vol. 1, No.1, November, 2010

[19] Shiping Zhu,Liang Yu,Kamel Bellouata.An improved fractal image coding algorithm based on adaptive threshold for quadtree partition. The International Society for Optical Engineering, v 7129, 2008

[20] Jinjiang Li, Da Yuan, Qingsong Xie, Caiming Zhang. Fractal Image Compression by Ant Colony Algorithm. The 9th International Conference for Young Computer Scientists

[21] B.Hurtgen,C.Stiler,Fast hierarchical codebook search for fractal coding of still images[J],Proceedings of EOS/SPIE Visual Communications PACS Medical Applications'93,Berlin, 1993:397 408.

[22] Victor De Lima, William R. S., Helio Pedrini, "Fast low Bit-Rate 3D Searchless Fractal Video Coding", IEEE 24th SIBGRAPI, pp. 189196, 2011.

[23] ZhenYao, RolandWilson, "Hybrid Fractal Video Coding With Neighbourhood Vector Quantisation", IEEE Proceedings, Data Compression Conference, UK, 2004.

[24] Meiqing Wang, Choi-Hong Lai, "A Hybrid Fractal Video Compression Method", Elsevier, International Journal of Computers and Mathematics with Applications 50, pp. 611-621, 2005.

[25] Meiqing Wang, Rong Liu, Choi-Hong Lai, "Adaptive Partition and Hybrid Method in Fractal Video Compression", Elsevier Inc. Computers and Mathematics with Applications, Vol. 51, pp. 17151726, 2006 